\title{
Low-load resistance training and blood flow restriction improves strength, muscle mass and functional performance in postmenopausal women: a controlled randomized trial
}

\begin{abstract}
Background: Evidence on the effectiveness of low-intensity resistance training and blood flow restriction (LRT-BFR) in postmenopausal women is scarce.

Objective: to analyze and compare the effects of high-load resistance training (HRT) and LRT-BFR with reduced pressure of blood flow restriction on maximal dynamic force (1RM), cross-sectional area of quadriceps (CSAq) and functional performance in postmenopausal women.

Methods: Twenty-four postmenopausal women (63.1 \pm 5.2 years) were randomly allocated into three groups: LRT-BFR $(n=9)$; HRT $(n=9)$ and control $(\mathrm{CON} ; n=6)$. 1 RM, CSAq, Rising from the floor (RFF), sit and stand up (SSU) and timed up and go test (TUGT) were applied before, after 8, and 16 weeks of resistance training.

Results: After 16 weeks, there were significant $(\mathrm{p}<0.05)$ and similar changes $(\mathrm{p}>0.05)$ for LRT-BFR and HRT for 1RM and CSAq, TUGT and SSU. RFF significantly decreased with LRT-BFR $(\mathrm{p}<0.001)$.

Conclusions: Both HRT and LRT-BFR improved 1RM, CSAq, and functional performance in postmenopausal women.
\end{abstract}

Keywords: functionality, hypertrophy, ischemia, lean mass, sarcopenia
Volume 4 Issue 2 - 2019

\author{
Piettra Moura Galvão Pereira,' Amandio \\ Aristides Rihan Geraldes, ${ }^{2}$ Maria da Glória \\ David Silva Costa, ${ }^{3}$ Joamira Pereira de \\ Araújo, ${ }^{4}$ Rodrigo Ramalho Aniceto, ${ }^{5}$ Manoel \\ da Cunha Costa, ${ }^{6}$ Gilberto Cândido \\ Laurentino, ${ }^{7}$ Maria do Socorro Cirilo-Sousa ${ }^{8}$ \\ 'Doctor, Department of physical education, Federal University \\ of Paraiba, CESMAC University Center, Brazil \\ ${ }^{2}$ Doctor, Department of physical education, Federal University \\ of Alagoas, Brazil \\ ${ }^{3}$ Graduate, Department of physical education, Federal University \\ of Alagoas, Brazil \\ ${ }^{4}$ Master, Federal University of Paraíba, Brazil, and Federal \\ Institute of Science and Technology of Ceará, Brazil \\ ${ }^{5}$ Doctor, Federal University of Paraiba, and Federal Institute of \\ Science and Technology of Rio Grande do Norte, Brazil \\ ${ }^{6}$ Doctor, University of Pernambuco, Brazil \\ Doctor, University of São Paulo, Brazil \\ ${ }^{8}$ Doctor, Department of physical education, Federal University \\ of Paraíba, Brazil
}

\begin{abstract}
Correspondence: Piettra Moura Galvão Pereira,Doctor, Department of physical education, Federal University of Paraíba, CESMAC University Center, Brazil, Tel +5582 99666-9116, Email piettra_galvao@hotmail.com
\end{abstract}

Received: February 15, 2019 | Published: March 05, 2019

\section{Introduction}

Changes in hormonal status related to menopause exert negative effects on muscle mass and strength, ${ }^{1}$ and may influence physical fitness and functional performance. Resistance training has been shown to maintain or increase strength, ${ }^{2}$ muscle mass, ${ }^{3}$ and functional performance ${ }^{4}$ when using intensities greater than $65 \%$ of maximal repetition (1RM). ${ }^{5}$ However, high intensities may not be well tolerated by frail elderly or those with joint problems. Thus, resistance training performed with loads between 20 and $50 \%$ of $1 \mathrm{RM}$ combined with blood flow restriction (LRT-BFR) may be an efficient strategy to promote neuromuscular adaptations similar to high-load resistance training (HRT) without BFR.

However, specifically in postmenopausal women, researches on the effect of LRT-BFR on strength, muscle mass, and functional performance are scarce. ${ }^{6-9}$ However, in Thiebaud et al. ${ }^{8}$ training was performed with elastic bands and in Araújo et al. ${ }^{7}$ in an aquatic environment. To use LRT-BFR, it is necessary to increase the safety in the application of the method to avoid potentially negative physiological factors, such as, for example, reduction of nerve conduction velocity, thrombus formation or induction to microvascular occlusion and discomfort ${ }^{9}$, especially in the presence of hypertension, diabetes and endothelial dysfunction, a frequent condition in postmenopausal women. ${ }^{10}$
In this study, it was hypothesized that in LRT-BFR, the application of moderate restrictive pressure (RP) during exercise associated with the release of pressure at low levels during rest among series could induce gains similar to those of HRT and would minimize risks and discomfort related to training, favoring adherence. Thus, the aim of this study was to analyze the effects of an LRT-BFR protocol, with reduced RP, on maximum dynamic force, cross-sectional area of the femoral quadriceps and functional performance in postmenopausal women. Additionally, compare the effects of this type of training with HRT on the same variables.

\section{Methods}

A randomized, open label, controlled trial with two parallel arms was performed. With the approval of local Research Ethics Committee (Protocol no. 817.007) the study included twenty-four women (63.1 \pm 5.2 years, $66.1 \pm 8.5 \mathrm{~kg}, 153.7 \pm 7.4 \mathrm{~cm})$ physically active, ${ }^{11}$ functionally independent, with central systolic blood pressure, pulse wave velocity and normal ankle brachial index, ${ }^{12-14}$ who did not exercise with weights in the previous six months. Participants who missed three or more consecutive sessions and/or did not attend $75 \%$ of training sessions were excluded. Figure 1 depict the flowchart CONSORT diagram.

All participants received information about the benefits and 
possible risks related to the research and signed the informed consent form. The study was approved by local Research Ethics Committee No. 817.007, and by Brazilian Registry of Clinical Trials: RBR4hqbmf (Figure 1).

\section{Experimental design}

Figure 2 shows the experimental design and the order for each of the measurements performed during the experimental period.

Participants were balanced by the estimated 1RM values and then randomized for the secret allocation into groups with randomizer.org. At the end of intervention, HRT ( $\mathrm{n}=9$ of 11), LRT-BFR ( $\mathrm{n}=9$ of 10$)$ and control groups (CON; $n=6$ of 10) participated in the analyses. The HRT group performed three series of ten repetitions, with a 60 -s interval between series and load of $70 \%$ of 1 RM, ${ }^{5}$ while the LRT-BFR group performed four series of 15 repetitions, with a 30-s interval between series, with load of $30 \%$ of $1 \mathrm{RM}$. The pressure applied to the cuff for the training was $50 \%$ of $\mathrm{RP}$ (mean $\pm \mathrm{SD}$ : right leg [ $83.94 \pm 16.12$ $\mathrm{mmHg}]$, left leg [85.83 $\pm 19.94 \mathrm{mmHg}]$ ); however, during the recovery intervals between series, cuffs were deflated and maintained at 50 $\mathrm{mmHg}$. The CON group did not perform the investigated exercise, free squat with bar. Cadence of two seconds for each phase of the movement was adopted (digital metronome; Korg, MA-30, Brazil).

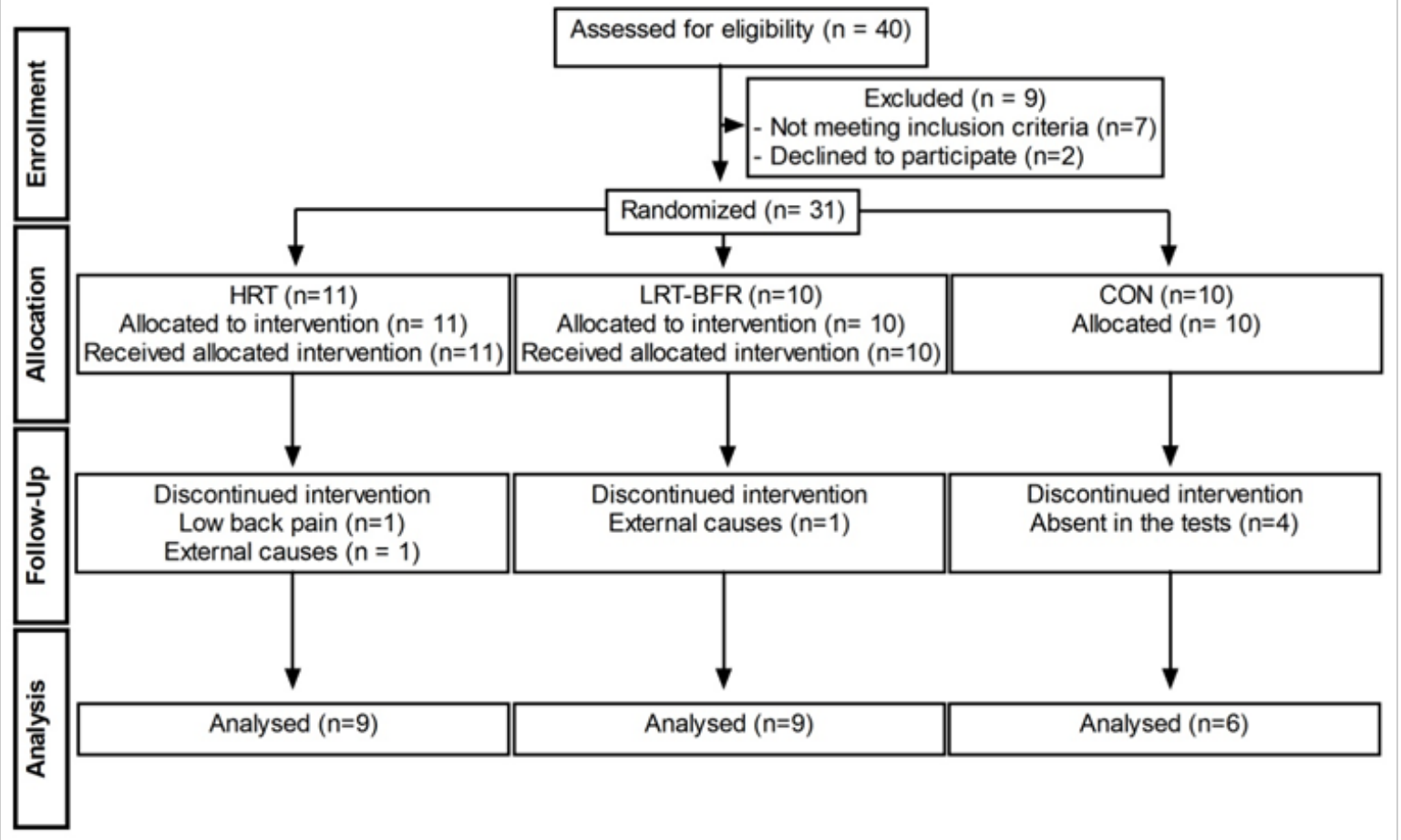

Figure I CONSORT diagram showing the flow of participants through the study.

TRAINING WEEKS

\begin{tabular}{|c|c|c|c|c|c|c|c|c|c|c|c|}
\hline & \multicolumn{5}{|c|}{ PRE } & \multirow[b]{2}{*}{ 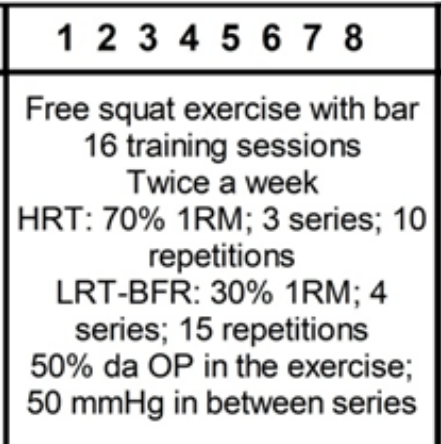 } & \multicolumn{2}{|c|}{ INTER } & \multirow[b]{2}{*}{$\begin{array}{c}910111213141516 \\
\text { Free squat exercise with bar } \\
16 \text { training sessions } \\
\text { Twice a week } \\
\text { HRT: } 70 \% \text { 1RM; } 3 \text { series; } 10 \\
\text { repetitions } \\
\text { LRT-BFR: } 30 \% 1 \mathrm{RM} ; 4 \\
\text { series; } 15 \text { repetitions } \\
50 \% \text { da OP in the exercise; } \\
50 \text { mmHg in between series }\end{array}$} & \multicolumn{2}{|c|}{ POST } \\
\hline 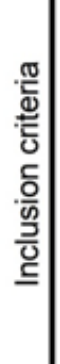 & 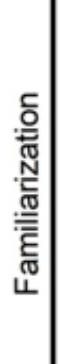 & 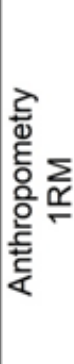 & 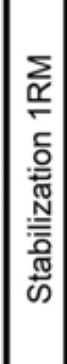 & 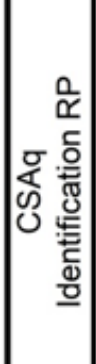 & 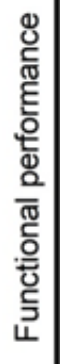 & & 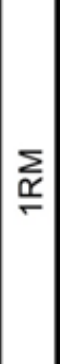 & 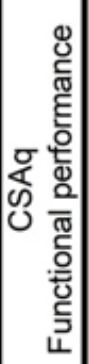 & & $\underset{\substack{\underline{x} \\
\Sigma}}{\sum}$ & 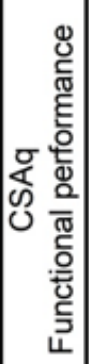 \\
\hline & & & & & & & & & & nterva & \\
\hline
\end{tabular}

Figure 2 Experimental timeline. IRM: One maximum repetition; CSAq: femoral quadriceps cross-sectional area; HRT: high-load resistance training; LRT-BFR: low-load resistance training and blood flow restriction; OP: occlusion pressure. 
Before starting the session, warm-up with a five-minute walk was performed, followed by 10 repetitions of squat with no additional load. To motivate participants, after squat exercises, all groups performed three series of 10 repetitions of exercises of elbow flexion and extension and shoulder abduction with dumbbell, ending with 10 minutes of stretching.

To ensure safety, promote adaptation and adherence to training, from the 1 st to the 8 th weeks, HRT and LRT-BFR trained with constant loads. From the 9th to the 16th week, training loads were increased by $1 \mathrm{~kg}$ when the participant was able to perform two repetitions in addition to those prescribed in the last series. Participants were evaluated before (PRE), after eight weeks (INTER) and sixteen weeks (POST). CON participants were evaluated at PRE and POST intervention.

\section{Maximum dynamic strength}

The maximum dynamic strength was estimated with the one repetition maximum test in the free squat exercise with bar, with the same cadence as the training. To increase the estimation accuracy, a maximum of seven repetitions was allowed. The load required to perform 1RM was estimated using the Wathen equation. ${ }^{15}$ Tests were repeated after $72 \mathrm{~h}$ until the difference between measurements was no more than $1 \mathrm{~kg}$. Tests were performed by a single trained evaluator, ICC of 0.98

\section{Femoral quadriceps cross-sectional area}

CSAq was performed on the thigh of the dominant limb by means of computed tomography (Toshiba Aquilion 64, Japan), as previously described. ${ }^{16}$ The analyzed image was the closest to the midpoint between the greater trochanter of the femur to the inferior border of the lateral epicondyle of the femur through the Aquilion software (Toshiba Aquilion 64, Japan). All measurements were performed by a single evaluator, $\mathrm{ICC}=0.99$.

\section{Arterial occlusion pressure determination}

The arterial occlusion point $(\mathrm{mmHg})$ was identified with a vascular doppler (DV 610B, MedMega, Brazil) as previously described. ${ }^{7}$ Briefly, with the participants in a supine position an adapted sphygmomanometer $(18 \mathrm{~cm}$ wide $\times 80 \mathrm{~cm}$ long $)$ was placed on the most proximal portion of both thighs and inflated to the point of arterial occlusion.

\section{Functional performance tests}

Functional performance was assessed through the following tests: a) Rising from the floor (RFF) as described by Alexander et al. ${ }^{17}$; b) sit and stand up (SSU) according to the Bohannon ${ }^{18}$ and c) Timed Up and Go Test (TUGT) previously described in Araújo et al. ${ }^{7}$ All tests were performed by a single evaluator trained using a digital timer (JS307-Junsd ${ }^{\circledR}$-Brazil). ICC values of 0.93, 0.86, 0.88 were observed for RFF, SSU and TUGT, respectively.

\section{Statistical analysis}

Data normality and homogeneity were evaluated by the ShapiroWilk and Levene test. Variable 1RM had no normal distribution, so a logarithmic transformation was made. Repeated measures ANOVA for factors time (PRE, INTER and POST) and group (HRT and LRTBFR) was used to evaluate the results of 1RM, CSAq measurements and functional performance (RFF, SSU and TUGT). The Bonferroni post hoc test was used when the $\mathrm{F}$ statistic presented statistical significance, set at $\mathrm{p}<0.05$. The effect size (ES) with $\eta_{p}^{2}$ was used for multiple comparisons and with Cohen's $d$ for the comparisons between pairs. ${ }^{19}$

To guarantee the validity of results, reproducibility analysis $(\mathrm{n}=10)$ was performed by ICC. The sample size was estimated a priori to detect differences (power of $0.90, \alpha$ of 0.05 , ES of 0.26 and correlation of 0.75 ) in 1RM force in squatting with data from a pilot study. Thus, a minimum of 24 participants was necessary to compose the three groups. The $\mathrm{G}^{*}$ Power statistical software version 3.0.10 and SPSS 20.0 were used.

\section{Results}

There was no significant difference among groups at PRE intervention time $(\mathrm{p}>0.05)$ for all variables.

\section{Maximum dynamic force}

In the multiple comparisons, there was a significant effect of time $(\mathrm{p}<0.001 ; \mathrm{ES}=0.98)$ and significant time $\mathrm{x}$ group interaction $(\mathrm{p}$ $<0.001 ; \mathrm{ES}=0.55$ ). HRT group differed significantly from INTER to POST; ES:0.78 [-0.149; -0.034], $\mathrm{p}=0.004$ and from PRE to POST; ES: 1.21 [-0.181;-0.101], $\mathrm{p}<0.001$, while LRT-BFR differed from PRE to POST; ES: 0.53 [-0.139; -0.060$], \mathrm{p}<0.001$. There was no significant difference among experimental groups (HRT and LRT-BFR); however, both differed from the CON group at POST (Figure 3A).

\section{Femoral quadriceps cross-sectional area}

CSAq showed a significant effect of time $(\mathrm{p}<0.001$; $E S=0.97)$ and significant time $\mathrm{x}$ group interaction $(\mathrm{p}<0.001$; $\mathrm{ES}=0.53)$. Both HRT; ES:0.28 [-3.927;-0.530], $\mathrm{p}=0.013$ and LRT-BFR groups; ES:0.34 [-5.506; -2.108], $\mathrm{p}<0.001$, differed significantly from PRE to POST. There was no significant difference among groups at any time (Figure 3B).

\section{Functional performance}

There was a significant effect of time $(p<0.00 ; E S \geq 0.55)$ and significant time $\mathrm{x}$ group interaction for all functional tests $(\mathrm{p}<0.001$; $\mathrm{ES} \geq 0.45$ ). There was no significant difference among experimental groups HRT and LRT-BFR; however, both differed from the CON group in RFF, SSU and TUGT tests at POST (Figure 4).

There was a reduction in the RFF execution time only for the LRTBFR group from PRE to POST; ES: -0.69 [0.733;2.049], $\mathrm{p}<0.001$ (Figure 4A). Significant increases in the number of repetitions in the SSU test were observed in the HRT group from PRE to INTER; ES:1.21 [-4.873;-1.127], $\mathrm{p}=0.003$ and from PRE to POST; ES: 2.01 [-6.826;-3.174], $\mathrm{p}<0.001$, while in the LRT-BFR group, significant differences were observed from INTER to POST; ES: 1.81 [-6.656;2.233], $\mathrm{p}<0.001$ and from PRE to POST; ES:1.77 [-6.937;-3.285], p $<0.001$ (Figure 4B).

In relation to the TUGT test, there was a significant difference in the HRT group from PRE to POST; ES:-0.73 [0.075; 1.179], $\mathrm{p}=0.028$, while in the LRT-BFR group, there was a significant difference from INTER to POST;ES:-2.04 [0.726;1.830], $\mathrm{p}<0.001$ and from PRE to POST; ES: -2.04 [0.726; 1.830], $\mathrm{p}<0.001$ (Figure 4C). There was no change in the CON group from PRE to POST test for any of the variables studied. 
a

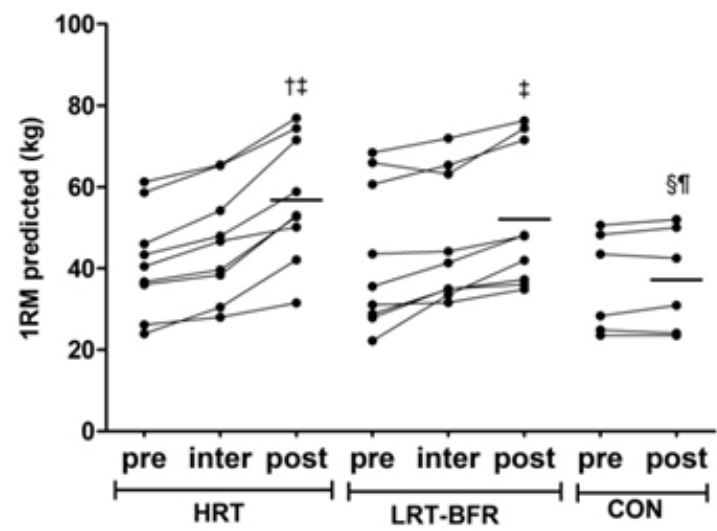

b

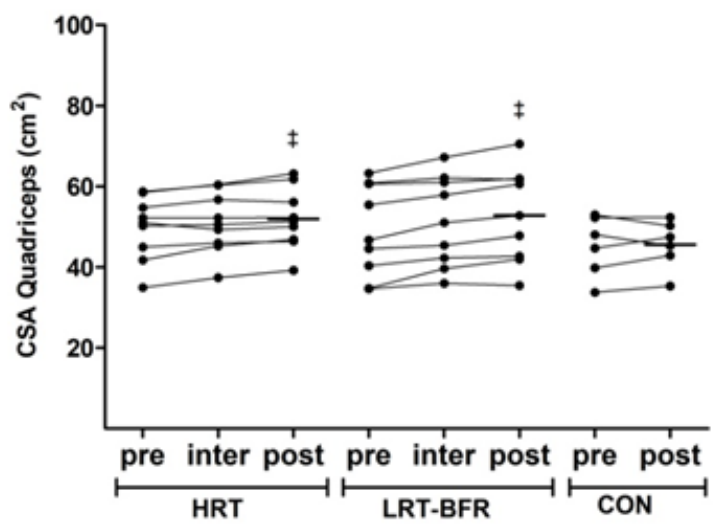

Figure $3 \mathrm{~A}$ ) Strength to perform maximum repetition and B) femoral quadriceps cross-sectional area in groups: high load (HRT), low load with blood flow restriction (LRT-BFR) and control group (CON) at conditions pre (PRE), after 8 (INTER) and I6 weeks (POST). † Significant difference from INTER; $\ddagger$ Significant difference from PRE; $§$ Significant difference from HRT; $\uparrow$ Significant difference from LRT-BFR. $\mathrm{p}<0.05$.
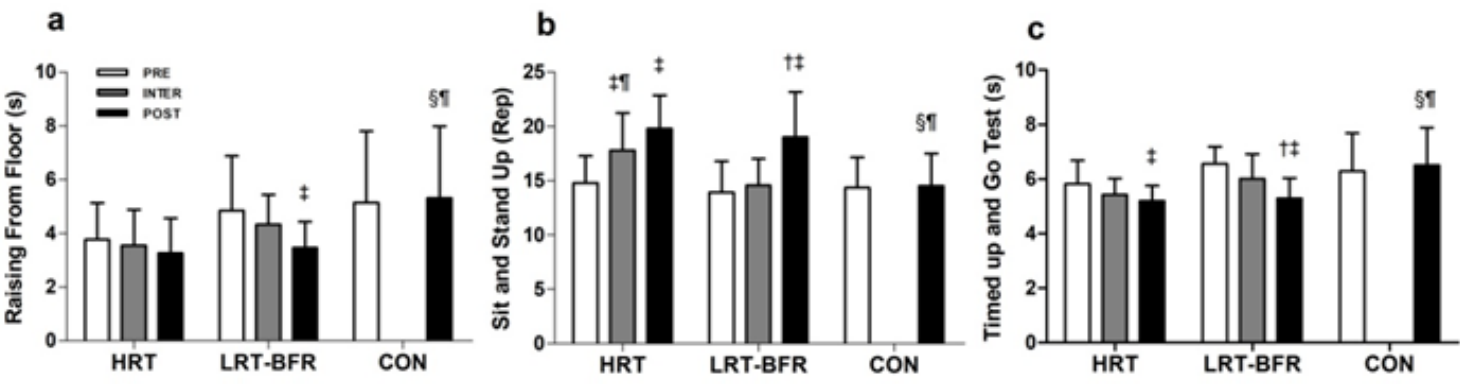

Figure 4 Functional performance tests:A) rising from the floor; B) sit and stand up; C) timed up and go test, groups: high load (HRT), low load with blood flow restriction (LRT-BFR) and control (CON) in conditions before (PRE), after 8 (INTER) and I6 weeks (POST). † Significant difference from INTER; $\ddagger$ Significant difference from PRE; $§$ Significant difference from HRT; $\uparrow$ Significant difference from LRT-BFR. $\mathrm{P}<0.05$

\section{Discussion}

To our knowledge, this was the first study to investigate the effects of an LRT-BFR protocol that, in addition performed with low RP values, partially released limb compression during recovery intervals on functional performance, 1RM and CSAq in postmenopausal women. The main findings of this study were: 1) both HRT and LRT-BFR promoted significant increases in functional performance for TUGT and SSU, 1RM strength and CSAq; 2) only the LRT-BFR group showed significant improvements for RFF; 3) HRT and LRTBFR differed significantly from CON in RFF, SSU, TUGT and 1RM.

The efficacy of HRT to induce adaptations in strength and muscle mass in the elderly is well established in literature. ${ }^{2,3}$ Regarding LRTBFR, similarly to the present study, Thiebaud et al. ${ }^{8}$ Araújo et al. ${ }^{7}$ Yasuda et al. ${ }^{20.21}$ Yokokawa et al..$^{22}$ and Karabulut et al..$^{23}$ observed significant increases $(p<0.001)$ in the $1 R M$ strength in exercises involving the lower limbs in older men and women. However, Vechin et al. ${ }^{24}$ observed no significant differences in $1 \mathrm{RM}$ after an exercise program with LRT-BFR $(\mathrm{p}=0.067)$. Among the studies cited, only Thiebald et al. ${ }^{8}$ and Araújo et al. ${ }^{7}$ investigated a sample specifically of postmenopausal women; however, methodological procedures were different from the present study, as Thiebald et al. ${ }^{8}$ used elastic bands as resistance and Araújo et al..$^{7}$ performed exercises in the aquatic environment. Like the present study, Vechin et al. ${ }^{24}$ observed no differences between HRT and LRT-BFR.

According to Yasuda et al. ${ }^{20,25}$ and Vechin et al. ${ }^{24}$ there was a significant difference of CSAq in POST. Such adaptations demonstrate important clinical relevance when moderate effect sizes are observed, since the adaptive capacity of muscle tissue in menopausal women is able to minimize the debilitating effects of sarcopenia.

Differently from Henwood and Taaffe $^{26}$ no improvement in performance for RFF with HRT was observed in the present study. In LRT-BFR, there was a significant reduction in time for RFF at POST (Figure $3 \mathrm{~A}$ ) with a very high effect size. It is assumed that the best performance in RFF was influenced by the free squat exercise used in the intervention, since it is multi-articular and involves the largest muscle groups of the lower limbs and resembles activities of the daily living such as rising from the floor or from the chair. However, no investigation was found on the effect of LRT-BFR on RFF.

In the SSU test, there was a significant difference between HRT and LRT-BFR groups at INTER; however, the difference was not maintained after the end of intervention (Figure 3B). Since muscle strength and power are determinants of performance in the SSU test, the influence of muscle activation is determinant for rapid force development, ${ }^{27}$ and based on the $1 \mathrm{RM}$ and CSAq results, there is evidence $^{28}$ that the force gain in HRT had predominance of the neural contribution, while in LRT-BFR, it was due to intrinsic muscular factors such as the composition of the myosin heavy chain and the cross-sectional area of muscle fiber. ${ }^{29}$ At POST, HRT and LRT-BFR groups showed significant improvements in SSU such as Yasuda et al. ${ }^{20}$

Similar to the effects of LRT-BFR on TUGT performance observed in the present study (Figure 3C), Yokokawa et al..$^{22}$ and Araújo et al. ${ }^{7}$ observed significant differences at POST. 
High and continuous RP contributes to early muscle fatigue; however, before fatigue is achieved, muscle activation levels are similar when compared to intermittent RP use $;^{30}$ however, in most studies, ${ }^{20,21,23,25}$ high RP values were used and maintained throughout the exercise. In the elderly, only one study used RP of $50 \%$ of the occlusion pressure; ${ }^{24}$ however, it was maintained in intervals between series. Regarding tolerance to training discomfort, it is important to point out that among HRT participants test interruption due to discomfort, while for the LRT-BFR group, there was no test interruption due to this reason.

Some limitations may be highlighted in the present study, such as the lack of diet control of study participants. However, studies ${ }^{20,21,23,25}$ showed positive effects on strength and hypertrophy gains with HRT and LRT-BFR strategies in older adults without dietary control.

The results of the present study indicate that the protocol used for low-load resistance training and blood flow restriction, with wider cuffs, moderate restrictive pressure $(50 \%$ of the arterial occlusion point) and pressure release between series up to one a fixed point of $50 \mathrm{mmHg}$ can be used as an alternative method to training with high loads, since it was able to favor the metabolic accumulation necessary to trigger the physiological chain to produce increased muscle strength, femoral quadriceps cross-sectional area and improve functional performance.

\section{Acknowledgments}

The authors thank volunteers who participated in the study. They also thank the Heart Hospital of Alagoas that provided the Applanation Tonometry examinations and the University Hospital of the Federal University of Alagoas for the Computed Tomography examinations.

\section{Conflicts of interest}

The authors declare no conflict of interest.

\section{References}

1. Maltais ML, Desroches J, Dionne IJ. Changes in muscle mass and strength after menopause. J Musculoskelet Neuronal Interact. 2009;9(4):186-197.

2. Daniels P, Burns RD, Brusseau TA, et al. Effect of a randomised 12-week resistance training programme on muscular strength, cross-sectional area and muscle quality in women having undergone Roux-en-Y gastric bypass. J Sports Sci. Routledge. 2017;36:529-35.

3. Bechshøft RL, Malmgaard CNM, Gliese B, et al. Improved skeletal muscle mass and strength after heavy strength training in very old individuals. Exp Gerontol. 2017;92:96-105.

4. Dias CP, Toscan R, de Camargo M, et al. Effects of eccentric-focused and conventional resistance training on strength and functional capacity of older adults. Age (Omaha). 2015;37:99.

5. Wojtek JCZ, David NP, Maria AFS, et al. ACSM. Exercise and physical activity for older adults. Med Sci Sports Exerc. 2009;41:1510-30.

6. Takarada Y, Takazawa H, Sato Y, et al. Effects of resistance exercise combined with moderate vascular occlusion on muscular function in humans. J Appl Physiol. 2000;88(6):2097-2106.

7. Araújo JP, Neto GR, Loenneke JP, et al. The effects of water-based exercise in combination with blood flow restriction on strength and functional capacity in post-menopausal women. Age (Omaha). 2015;37:110
8. Thiebaud RS, Loenneke JP, Fahs CA, et al. The effects of elastic band resistance training combined with blood flow restriction on strength, total bone-free lean body mass and muscle thickness in postmenopausal women. Clin Physiol Funct Imaging. 2013;33:344-352.

9. Loenneke JP, Wilson JM, Wilson GJ, et al. Potential safety issues with blood flow restriction training. Scand J Med Sci Sports. 2011;21(4):510 518 .

10. Gierach GL, Johnson BD, Merz CNB, et al. Hypertension, menopause, and coronary artery disease risk in the women 's ischemia syndrome evaluation (WISE) study. J Am Coll Cardiol. 2006;47:S50-58.

11. Tudor LC, Craig CL, Thyfault JP, et al. A step-defined sedentary lifestyle index: <5000 steps/day. Appl Physiol Nutr Metab. 2013;38(2):100-114.

12. Williams B, Lacy PS, Thom SM, et al. Differential impact of blood pressure - lowering drugs on central aortic pressure and clinical outcomes: principal results of the conduit artery function evaluation ( CAFE ) study. Circulation. 2006;113:1213-1225.

13. Resnick HE, Lindsay RS, McDermott MM, et al. Relationship of high and low ankle brachial index to all-cause and cardiovascular disease mortality: the strong heart study. Circulation. 2004;109:733-739.

14. European Society of Cardiology. Determinants of pulse wave velocity in healthy people and in the presence of cardiovascular risk factors: establishing normal and reference values. Eur Heart J. 2010;31:23382350

15. Wathen D. Load assignment. In: Baechle TR, editor. Essentials Strength Train Cond. Champaign. 1994;435-446.

16. Visser M, Goodpaster BH, Kritchevsky SB, et al. Muscle mass, muscle strength, and muscle fat infiltration as predictors of incident mobility limitations in well-functioning older persons. J Gerontol A Biol Sci Med Sci. 2005;60(3):324-333.

17. Alexander N, Ulbrich J, Raheja A, et al. Rising from the floor in older adults. J Am Geriatr Soc. 1997;45:564-569.

18. Bohannon R. Sit-to-stand test for measuring performance of lower extremity muscles. Percep Mot Ski. 1995;80:163-166.

19. Cohen J. Statistical power analysis for the behavioral sciences. Lawrence E. Hillsdale, NJ; 1988

20. Yasuda T, Fukumura K, Fukuda T, et al. Effects of low-intensity, elastic band resistance exercise combined with blood flow restriction on muscle activation. Scand J Med Sci Sport. 2014;24(1):55-61.

21. Yasuda T, Fukumura K, Sato Y, et al. Effects of detraining after blood flow-restricted low-intensity training on muscle size and strength in older adults. Aging Clin Exp Res. 2014;26(5):561-564.

22. Yokokawa Y, Hongo M, Urayama H, et al. Effects of low-intensity resistance exercise with vascular occlusion on physical function in healthy elderly people. Biosci Trends. 2008;2(3):117-123.

23. Karabulut M, Abe T, Sato Y, et al. The effects of low-intensity resistance training with vascular restriction on leg muscle strength in older men. Eur J Appl Physiol. 2010;108(1):147-155.

24. Vechin FC, Libardi CA, Conceição MS, et al. Comparisons between low-intensity resistance training with blood flow restriction and highintensity resistance training on quadriceps muscle mass and strength in elderly. J Strength Cond Res. 2015;29:1071-1076.

25. Yasuda T, Fukumura K, Fukuda T, et al. Muscle size and arterial stiffness after blood flow-restricted low-intensity resistance training in older adults. Scand J Med Sci Sport. 2014;24(5):799-806

26. Henwood TR, Taaffe DR. Improved physical performance in older adults undertaking a short-term programme of high-velocity resistance training. Gerontology. 2005;51:108-115. 
27. Andersen LL, Aagaard P. Influence of maximal muscle strength and intrinsic muscle contractile properties on contractile rate of force development. Eur J Appl Physiol. 2006;96(1):46-52.

28. Moritani T, de Vries $\mathrm{H}$ a. Neural factors versus hypertrophy in the time course of muscle strength gain. Am J Phys Med. 1979;58(3):115-130.

29. Pearson SJ, Hussain SR. A Review on the mechanisms of blood-flow restriction resistance training-induced muscle hypertrophy. Sport Med. 2014;45(2):187-200.

30. Yasuda T, Loenneke JP, Ogasawara R, et al. Influence of continuous or intermittent blood flow restriction on muscle activation during low-intensity multiple sets of resistance exercise. Acta Physiol Hung. 2013;100(4):419-426. 